\title{
Determination of the coherence length and the Cooper-pair size in unconventional superconductors by tunnelling spectroscopy
}

\author{
A. Mourachkine \\ Nanoscience Centre and the Cavendish Laboratory, University of Cambridge, \\ 11 J. J. Thomson Avenue, Cambridge CB3 OFF, UK
}

(Dated 27 May 2004)

\begin{abstract}
The main purpose of the paper is to discuss a possibility of the determination of the values of the coherence length and the Cooper-pair size in unconventional superconductors by using tunnelling spectroscopy. In the mixed state of type-II superconductors, an applied magnetic field penetrates the superconductor in the form of vortices which form a regular lattice. In unconventional superconductors, the inner structure of a vortex core has a complex structure which is determined by the order parameter of the superconducting state and by the pairing wavefunction of the Cooper pairs. In clean superconductors, the spatial variations of the order parameter and the pairing wavefunction occur over the distances of the order of the coherence length and the Cooper-pair size, respectively. Therefore, by performing tunnelling spectroscopy along a line passing through a vortex core, one is able, in principle, to estimate the values of the coherent length and the Cooper-pair size.
\end{abstract}

PACS numbers: 74.25.Op; 74.25.Qt; 74.50.+r; 74.25.Jb

\section{INTRODUCTION}

Superconductivity requires the electron pairing and the onset of long-range phase coherence. These two physical phenomena are independent of one another. In the framework of the Ginzburg-Landau theory [1], the superconducting state is characterized by the order parameter $\Psi$. The coherence length $\xi_{G L}$ is the characteristic scale over which variations of $\Psi$ occur, for example, near a superconductor-normal metal boundary. Generally speaking, the coherence length is different from the size of the Cooper pairs, $\xi$, which is related to the wavefunction of a Cooper pair $\psi$. Furthermore, the coherence length depends on temperature, $\xi_{G L}(T)$, while the Cooper-pair size is temperature-independent, at least, in conventional superconductors. The coherence length diverges at $T \rightarrow T_{c}$, where $T_{c}$ is the critical temperature. For every superconductor, the knowledge of the values of the coherence length and the size of Copper pairs is important for the understanding of the underlying mechanism of superconductivity.

The superconducting state can be destroyed by a sufficiently strong magnetic field. The variation of the thermodynamic critical field $H_{c}$ with temperature for a typeI superconductor is approximately parabolic: $H_{c}(T) \simeq$ $H_{c}(0)\left[1-\left(T / T_{c}\right)^{2}\right]$, where $H_{c}(0)$ is the value of the critical field at absolute zero. For a type-II superconductor, there are two critical fields, the lower critical field $H_{c 1}$ and the upper critical field $H_{c 2}$. In applied fields less than $H_{c 1}$, the superconductor completely expels the field, just as a type-I superconductor does below $H_{c}$. At fields just above $H_{c 1}$, flux, however, begins to penetrate the superconductor in microscopic filaments called vortices which form a regular lattice, as shown in Fig. 1(a). Each vortex consists of a normal core in which the magnetic field is large, surrounded by a superconducting region, and can be approximated by a long cylinder with its axis parallel to the external magnetic field. Inside the cylinder, the superconducting order parameter is zero, as illustrated in Fig. 1(b). The radius of the cylinder is of the order of the coherence length $\xi_{G L}$. The supercurrent circulates around the vortex within an area of radius $\sim \lambda$, the penetration depth. The vortex state of a superconductor, predicted theoretically by Abrikosov [2], is also known as the mixed state. By increasing the magnitude of the applied magnetic field from $H_{c 1}$ to $H_{c 2}$, the distance between vortices decreases, becoming zero at $H_{c 2}$. At $H_{c 2}$, the field penetrates completely the superconductor, making it normal.

Probably, the best technique for the determination of the behaviour of the vortex cores in the bulk of type-II superconductors is muon spin rotation $(\mu \mathrm{SR})$ measurements [3]. However, $\mu \mathrm{SR}$ are not able to determine (i) the inner structure of the vortex cores and (ii) the local density of states of quasiparticle excitations. Generally speaking, there is no perfect technique: every technique has some disadvantages.

Tunnelling spectroscopy is an unique probe of the superconducting state in that it can, in principle, reveal the quasiparticle excitation density of states directly with high energy resolution [4]. By using a scanning tunnelling microscope (STM), one can obtain images of the vortex lattice in the mixed state of superconductors, and perform local spectroscopy inside and outside vortex cores [5-14]. At low magnetic field $H \sim H_{c 1}$, when the distance between vortices is large $\left(\gg \xi_{G L}\right)$, the variation of the local density of states inside and outside vortex cores is determined by the order parameter $\Psi$ and the pairing wavefunction of the Cooper pairs $\psi$. The spatial variations of $\Psi$ and $\psi$ occur over the distances of the order of $\xi_{G L}$ and $\xi$, respectively (in the clean limit). Therefore, by performing tunnelling spectroscopy along a line 

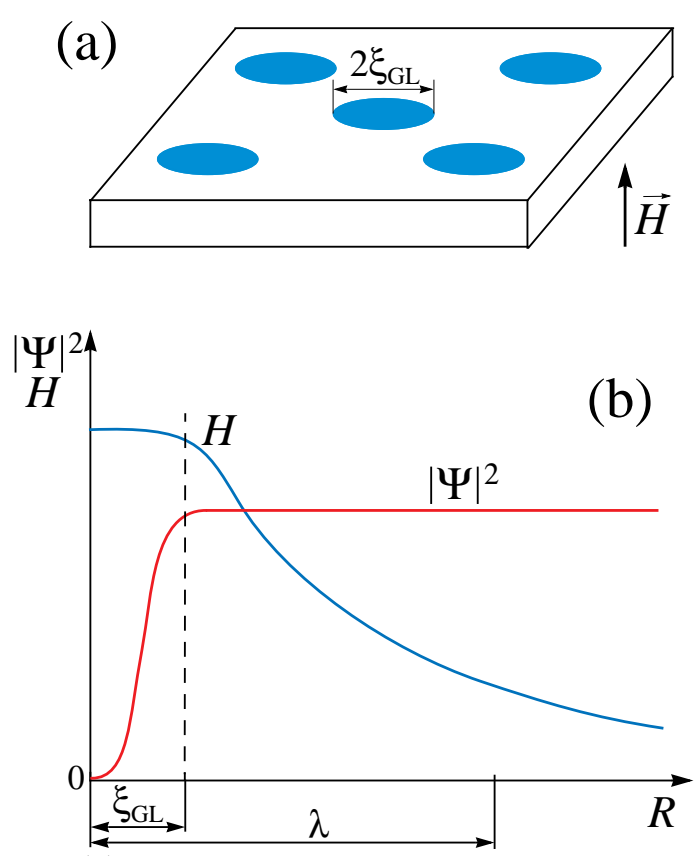

FIG. 1. (a) In the mixed state of type-II superconductors, an applied magnetic field penetrate the superconductor in the form of vortices which form a regular lattice. Each vortex consists of a normal core, and can be approximated by a long cylinder with its axis parallel to the external magnetic field. The radius of the cylinder is of the order of the coherence length $\xi_{G L}$. (b) Spatial variations of the magnetic field $H$ and the order parameter $\Psi$ inside and outside an isolated vortex in an infinite superconductor. $R$ is the distance from the center of the vortex, and $\xi_{G L}$ and $\lambda$ are the coherence length and the penetration depth of the superconductor, respectively (in type-II superconductors, $\xi_{G L}<\lambda$ ).

passing through the center of a vortex core, one can in principle estimate the values of the coherent length and the Cooper-pair size. The main purpose of the paper is to investigate a possibility of the determination of the values of the coherence length and the Cooper-pair size in unconventional superconductors by using tunnelling spectroscopy in the mixed state.

The paper is organized as follows. We first discuss the spatial variation of the local density of states in the vortex cores of conventional superconductors. Then, we shall turn our attention to half-conventional and unconventional superconductors. In the following section, we shall compare our results with real data obtained in halfconventional and unconventional superconductors. The paper ends with a discussion and conclusions.

\section{CONVENTIONAL SUPERCONDUCTORS}

Superconductivity requires the electron pairing and the onset of long-range phase coherence. In the framework of the BCS (Bardeen-Cooper-Schrieffer) theory for conventional superconductors [15], the electrons form

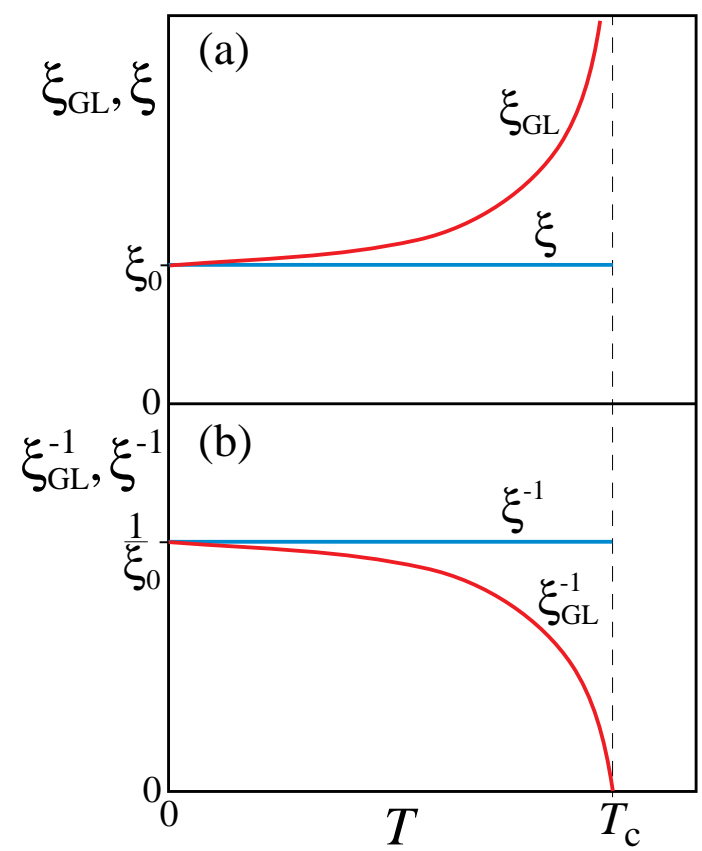

FIG. 2. Temperature dependences of (a) the coherence length $\xi_{G L}$ and the Cooper-pair size $\xi$, and (b) $1 / \xi_{G L}$ and $1 / \xi$ in "clean" conventional superconductors. In a first approximation, the Cooper-pair size is independent of temperature. The dependences $\xi_{G L}(T)$ and $1 / \xi_{G L}(T)$ are shown schematically.

pairs due to phonons, while the phase coherence is established by the overlap of the Cooper-pair wavefunctions. The latter process is also called the Josephson coupling. Such a way of the establishment of the longrange phase coherence gives rise to an order parameter which is a "magnified" version of the Cooper-pair wavefunctions. Therefore, the values of the coherence length and the Cooper-pair size in conventional superconductors coincide at $T=0: \xi_{G L}(0)=\xi(0)=\xi_{0}$, as shown in Fig. 2(a). The coherence length (or the Cooper-pair size) $\xi_{0}$ determined by the energy gap at zero temperature, $\Delta(T=0)$, is called intrinsic:

$$
\xi_{0}=\frac{\hbar v_{F}}{\pi \Delta(0)},
$$

where $v_{F}$ is the Fermi velocity (on the Fermi surface), and $\hbar=h / 2 \pi$ is the Planck constant. Let us estimate $\xi_{0}$. In a metal superconductor, $\Delta(0) \sim 1 \mathrm{meV}$. Substituting this value into Eq. (1), together with $v_{F} \approx 1.5 \times 10^{8} \mathrm{~cm} / \mathrm{s}$ and $\hbar=h / 2 \pi \simeq 6.5 \times 10^{-13} \mathrm{meV}$ s, we obtain $\xi_{0} \simeq 3 \times 10^{-5}$ $\mathrm{cm}=3 \times 10^{3} \AA$. Thus, the values of the intrinsic coherence length in conventional superconductors is very large in comparison with the interatomic distances $(\sim 1-2 \AA)$. In conventional superconductors, the electron pairing and the onset of phase coherence occur simultaneously at $T_{c}$. It is worth mention that Eq. (1) can be obtained in a good approximation by using the uncertainty principle $\delta p \xi_{0} \sim \hbar$, where $\delta p$ is the momentum spread [16]. In 
conventional superconductors, the assumption that $\xi(T)$ is constant with temperature is sufficiently good, because the $T_{c}$ value in conventional superconductors is very low and there are no structural transitions below $T_{c}$.

Consider the so-called clean and dirty limits for superconductors. In the clean limit, $\ell \gg \xi_{0}$, where $\ell$ is the electron mean free path; in the dirty limit, $\ell \ll \xi_{0}$. At $0<T<T_{c}$, the coherence length in "clean" conventional superconductors is always larger than the size of Cooper pairs, $\xi<\xi_{G L}(T)$, as depicted in Fig. 2(a). In the framework of the Ginzburg-Landau theory, the temperature dependence of the coherence length in "clean" superconductors at temperatures close to $T_{c}$ is given by

$$
\xi_{G L}^{c}(T)=0.74 \xi_{0}\left(1-\frac{T}{T_{c}}\right)^{-1 / 2} .
$$

For "dirty" superconductors, the Ginzburg-Landau temperature dependence of the coherence length at $T \sim T_{c}$ is

$$
\xi_{G L}^{d}(T)=0.85\left(\xi_{0} \ell\right)^{1 / 2}\left(1-\frac{T}{T_{c}}\right)^{-1 / 2} .
$$

From Eqs. (2) and (3), one can see that $\xi_{G L}^{c, d} \rightarrow \infty$ as $T \rightarrow T_{c}$. It is worth to mention that, in very "dirty" metals, the mean electron free path $\ell$ plays the role of the coherence length [17].

The condensation of the Cooper pairs in conventional superconductors, and therefore, of the condensate, occurs in momentum space. In this case, one can argue that the "real" characteristics of the superconducting condensate in conventional superconductors are not $\xi_{G L}$ and $\xi$ but $\xi_{G L}^{-1}$ and $\xi^{-1}$ shown in Fig. 2(b). The temperature dependence of $\xi_{G L}^{-1}$ in Fig. 2(b) is similar to the BCS temperature dependence of the energy gap, $\Delta(T)$, illustrating the increase of the phase stiffness with decreasing temperature.

Let us now discuss the variations of the characteristics of the superconducting state into the vortex cores appearing in the mixed phase of conventional superconductors. The spatial variations of the magnetic field and the order parameter inside and outside an isolated vortex are illustrated in Fig. 1(b). As shown by Gor'kov, the spatial variations of the energy gap in conventional superconductors are proportional to the variations of the order parameter, $\Delta \propto|\Psi|[18]$. Therefore, in conventional superconductors, the energy gap also goes to zero inside the vortex core, as shown in Fig. 3. In conventional superconductors, by performing tunnelling spectroscopy along a line passing through a vortex core, it is possible to estimate only the value of the coherence length $\xi_{G L}$ but not $\xi$. On the other hand, in conventional superconductors, $\xi \simeq \xi_{G L}$ at $T<T_{c} / 2$. As a consequence, the knowledge of $\xi_{G L}$ at low temperatures is sufficient.

Alternatively, the value of the coherence length can be estimated in momentum space. By performing topogra-

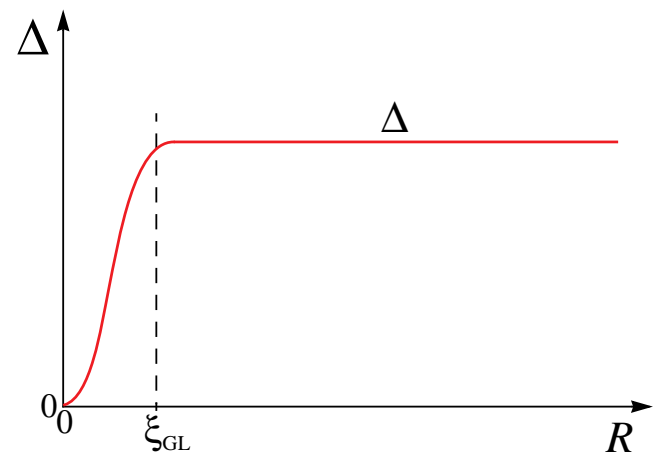

FIG. 3. Spatial variations of the energy gap $\Delta$ inside and outside an isolated vortex in conventional superconductors. $R$ is the distance from the center of a vortex core.

phy image above vortex cores at bias $|V|<\Delta / e$, where $e$ is the electron charge, and then, making the Fourier transform of the image, one can in principle estimate the value of the coherence length in accordance with the plot in Fig. 2(b).

In a "clean" conventional, s-wave superconductor, quasiparticles whose energy $E$ is less than the bulk energy gap, $E<\Delta$, may theoretically form discrete localized states in the vortex core $[19,20]$. Andreev reflections of quasiparticles from the normal core-superconductor interface give rise to these bound states. The lowest energy of the bound states is approximately $\sim \Delta^{2} / 2 E_{F}$, where $E_{F}$ is the Fermi energy [19]. In a superconductor with an order parameter having nodes, the bound state will "leak out" through the nodes, giving rise a broad peak at the Fermi level in the quasiparticle local density of states of vortex cores [21].

\section{HALF-CONVENTIONAL SUPERCONDUCTORS}

In this section we discuss the mixed phase in twoband superconductors. The Fermi surface of such superconductors consists of, at least, two disconnected sections. Below a certain temperature, the Cooper pairs are formed in one section of the Fermi surface due to the electron-phonon interaction. Then, due to either the interband scattering or Cooper-pair tunnelling, or both, the electron pairing is induced into the second section (band) of the Fermi surface. So, there are two types of the Cooper pairs in the system, and all of them are formed due to the electron-phonon interaction. Therefore, we shall call such superconductors as halfconventional. Consider a system in which the genuine Cooper pairs formed in the first band cannot establish alone the long-phase coherence. This can happen in a system in which the size of the Cooper pairs is small (in comparison with that of the Cooper pairs in metals) and their concentration is low. As a consequence, their wavefunctions do not overlap steadily. The long-range phase 


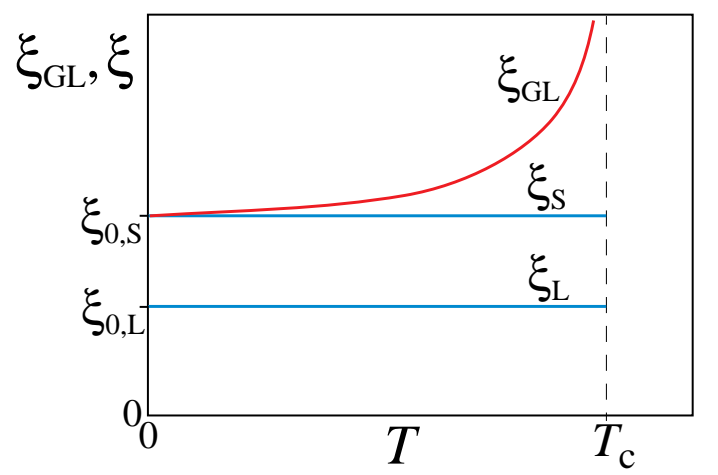

FIG. 4. Temperature dependences of the coherence length $\xi_{G L}$ and the Cooper-pair size $\xi_{L}$ and $\xi_{S}$ in "clean" two-band superconductors. In two-band superconductors, there two types of the Cooper pairs having the different size. In a first approximation, $\xi_{L}$ and $\xi_{S}$ are independent of temperature. The dependence $\xi_{G L}(T)$ is shown schematically.

coherence of the superconducting state appears owing to the Josephson coupling of the wavefunctions of the induced Cooper pairs. In reality, in two-band superconductors the genuine Cooper pairs are low-dimensional, i.e. one- or two-dimensional. At the same time, the induced Cooper pairs in the second band are three-dimensional.

Let us label the energy gap of the low-dimensional Cooper pairs by $\Delta_{L}$, and their size by $\xi_{L}$. Analogously, for the three-dimensional Cooper pairs, $\Delta_{S}$ and $\xi_{S}$. The labels "L" and "S" stand for "Large" and "Small", respectively, because always $\Delta_{L}>\Delta_{S}$. This is typical for induced superconductivity [17]. In contrast, $\xi_{L}<\xi_{S}$, i.e. the size of the three-dimensional Cooper pairs is always larger than that of the low-dimensional ones. This follows from the fact that $\xi \propto 1 / \Delta$ for any type of superconductivity. Denote the wavefunctions of the two types of the pairs by $\psi_{L}$ and $\psi_{S}$. The order parameter of the superconducting state $\Psi$ in the system is a "magnified" version of $\psi_{S}$, and the coherence length $\xi_{G L}$ of the order of $\xi_{S}$, as schematically shown in Fig. 4 .

We are going now to consider the variations of the characteristics of the superconducting state into the vortex cores appearing in the mixed state of "clean" halfconventional superconductors. Figure 5(a) shows the spatial variations of the order parameter and the averaged wavefunction $\left|\psi_{L}\right|$ into a vortex core. Inside a vortex core, the order parameter always goes to zero, while $\left|\psi_{L}\right|$ should not. Depending on the coupling strength of the low-dimensional Cooper pairs, there are three possible outcomes for the behaviour of $\left|\psi_{L}\right|$ into a vortex core, shown by the letters A, B and C in Fig. 5(a). The spatial variation of the averaged $\left|\psi_{L}\right|$ wavefunction, if such occurs, is of the order of $\xi_{L}$. From the discussion in the previous section, the smaller energy gap $\Delta_{S}$ will be always zero inside a vortex core, as depicted in Fig. 5(b). In contrast, the larger energy gap $\Delta_{L}$ should not be zero into a vortex core, and even, may remain unchanged [the

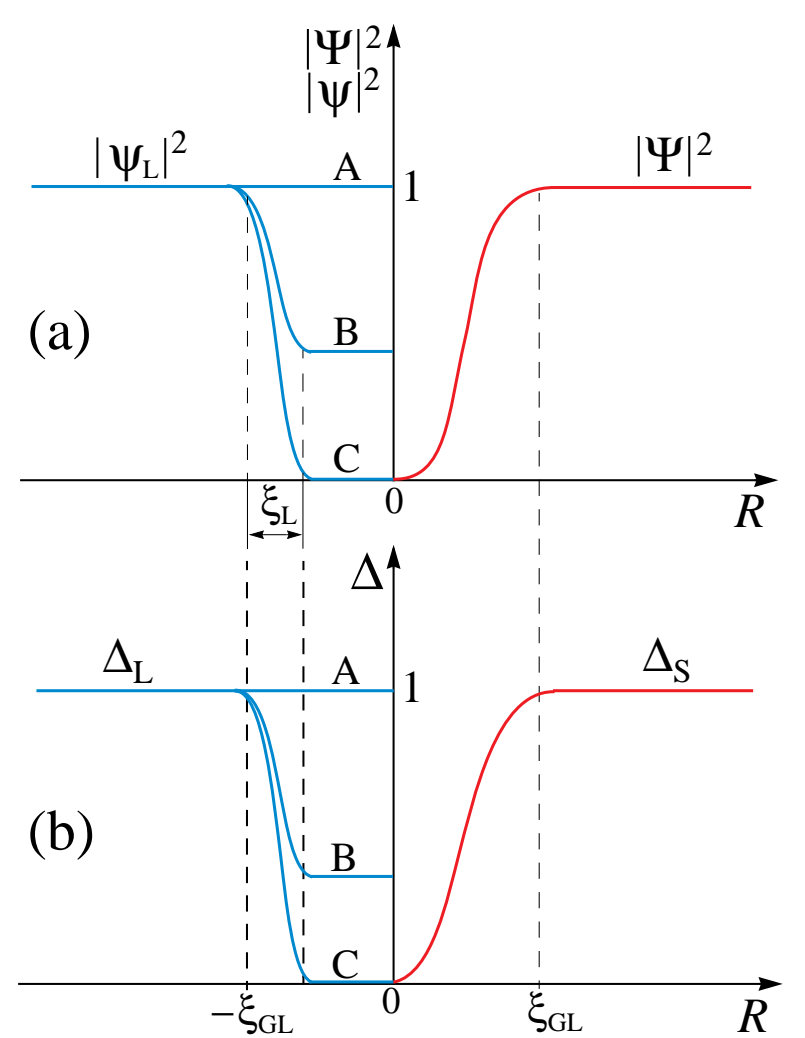

FIG. 5. Spatial variations of (a) the order parameter $|\Psi|^{2}$ and the averaged wavefunction $\left|\psi_{L}\right|^{2}$, and (b) the energy gaps $\Delta_{L}$ and $\Delta_{S}$ inside and outside an isolated vortex in an infinite "clean" two-band superconductor. $R$ is the distance from the center of the vortex. $\xi_{G L}$ is the coherence length, and $\xi_{L}$ is the size of the smaller (genuine) Cooper pairs $\left(\xi_{L}<\xi_{G L}\right)$. For simplicity, all the functions are normalized at $R=\infty$ to 1. Since the pairing wavefunction $\psi_{L}$ is not global, $\psi_{L}$ in plot (a) is average. The letters $\mathrm{A}, \mathrm{B}$ and $\mathrm{C}$ indicate a different behaviour of $\left|\psi_{L}\right|^{2}$ and $\Delta_{L}$.

case A in Fig. 5(b)]. In the latter case, the size of the Cooper pairs cannot be determined from the tunnelling data. Therefore, we discuss further only the cases $\mathrm{B}$ and $\mathrm{C}$ sketched in Fig. 5(b). The case $\mathrm{C}$ in Fig. 5 can theoretically occur; however, it is less likely that such a situation can be realized in practice because the existence of the induced Cooper pairs depends on the presence of the genuine Cooper pairs. In vortex cores of half-conventional superconductors, tunnelling measurements tuned to $\Delta_{S}$ should show the disappearance of the energy gap in the center of a vortex core, as illustrated in Fig. 6(a). Probing $\Delta_{L}$, one should observe its variation inside a vortex core sketched in Fig. 6(b). Since this variation of $\Delta_{L}$ occurs over the distance of the order of $\xi_{L}$, one can estimate the value of $\xi_{L}$ from the tunnelling data.

It is important to note that, in two-band superconductors in which the genuine Cooper pairs can along establish the long-range phase coherence, the magnitudes of both $\Delta_{S}$ and $\Delta_{L}$ will be zero in the center of a vortex core. Such a situation was discussed elsewhere [22]. To 


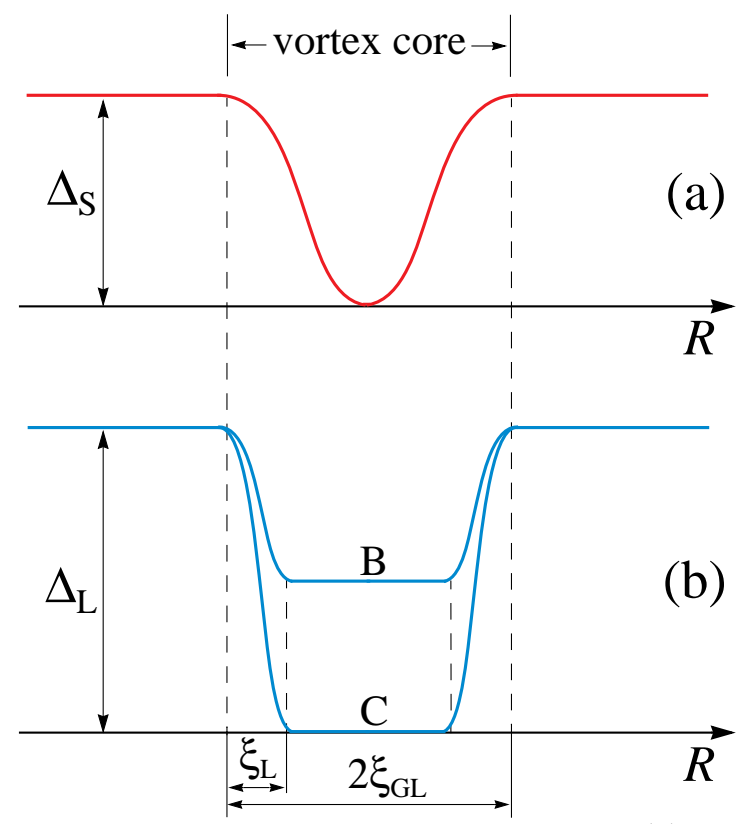

FIG. 6. Spatial variations of the energy gaps (a) $\Delta_{S}$ and (b) $\Delta_{L}$ inside and outside an isolated vortex in an infinite "clean" two-band superconductor. The line $R$ passes through the center of the vortex core. $\xi_{G L}$ is the coherence length, and $\xi_{L}$ is the size of the smaller (genuine) Cooper pairs $\left(\xi_{L}<\xi_{G L}\right)$. For $\Delta_{L}$, the letters B and $\mathrm{C}$ correspond to the cases shown in Fig. 5(b).

remind, we consider here two-band superconductors in which the genuine Cooper pairs formed in the first band cannot establish alone the long-phase coherence. The long-range phase coherence in this type of superconductors appears due to the overlap of the wavefunctions of the induced Cooper pairs in the second band.

As in the case of conventional superconductors, in halfconventional superconductors it is also not possible to estimate the value of $\xi_{S}$ from the tunnelling spectroscopy data. On the other hand, $\xi_{S} \approx \xi_{G L}$ at $T<T_{c} / 2$. For the estimation of the value of $\xi_{L}$, a hint can be suggested. It can be estimated from the following equation

$$
\xi_{L} \sim \xi_{G L} \frac{\Delta_{S}}{\Delta_{L}} .
$$

In half-conventional superconductors, the two energy gaps have both an s-wave symmetry typical for the electron pairing due to phonons. They are both anisotropic: if $\Delta_{S}$ is only slightly anisotropic, $\Delta_{L}$ is highly anisotropic, and can even have nodes. In halfconventional superconductors, the maximum magnitude of $\Delta_{L}$ is a few times $(\sim 3)$ larger than that of $\Delta_{S}$. This is typical for induced superconductivity [17].

\section{UNCONVENTIONAL SUPERCONDUCTORS}

Before we discuss the mixed phase of unconventional superconductors, let us first give the definition of an

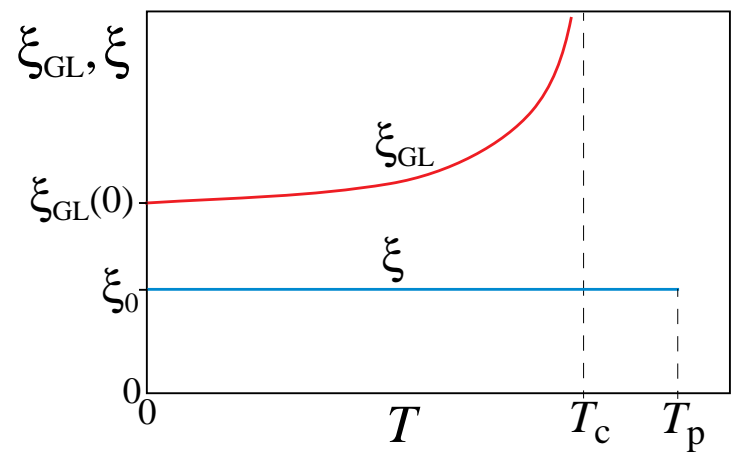

FIG. 7. Temperature dependences of the coherence length $\xi_{G L}$ and the Cooper-pair size $\xi$ in unconventional superconductors in which the superconducting condensate has a structure similar to that of a Russian doll (see text for more details). $T_{p}$ is the pairing temperature. In plot, $\xi$ is independent of temperature; however, in some cases, this assumption can be incorrect. The dependence $\xi_{G L}(T)$ is shown schematically.

unconventional superconductor. In a wide sense, a superconductor is unconventional if the mechanism of superconductivity in this superconductor is different from the BCS mechanism and from that of half-conventional superconductors. In this section, we shall consider two types of unconventional superconductors. We start with superconductors in which the superconducting condensate has a structure similar to that of a Russian doll.

\section{A. Russian-doll-like condensate}

In these superconductors, both the quasiparticle pairing and the long-range phase coherence are mediated by bosonic excitations. If the electron pairing is always mediated by bosonic excitations, this is not typical for the establishment of the phase coherence. In conventional and half-conventional superconductors, the long-range phase coherence occurs due to the overlap of the Cooperpair wavefunctions, thus, without participation of any bosonic excitations. In superconductors with a Russiandoll-like condensate, the electron pairing is mediated, for example, by phonons, and the phase coherence, for instance, occurs due to spin fluctuations. Assume that the Cooper pairs are formed above the critical temperature, $T_{p}>T_{c}$. In this type of unconventional superconductors, the symmetry of the Cooper-pair wavefunctions can differ from the symmetry of the order parameter of the superconducting condensate. Thus, the superconducting condensate in this type of superconductors has a structure similar to that of a Russian doll.

Figure 7 shows the temperature dependences of the coherence length $\xi_{G L}$ and the Cooper-pair size $\xi$ in unconventional superconductors with a Russian-doll-like condensate. For simplicity, assume that the Cooper-pair size is independent of temperature, or at lest, is a weak function of temperature. In real superconductors, if the 


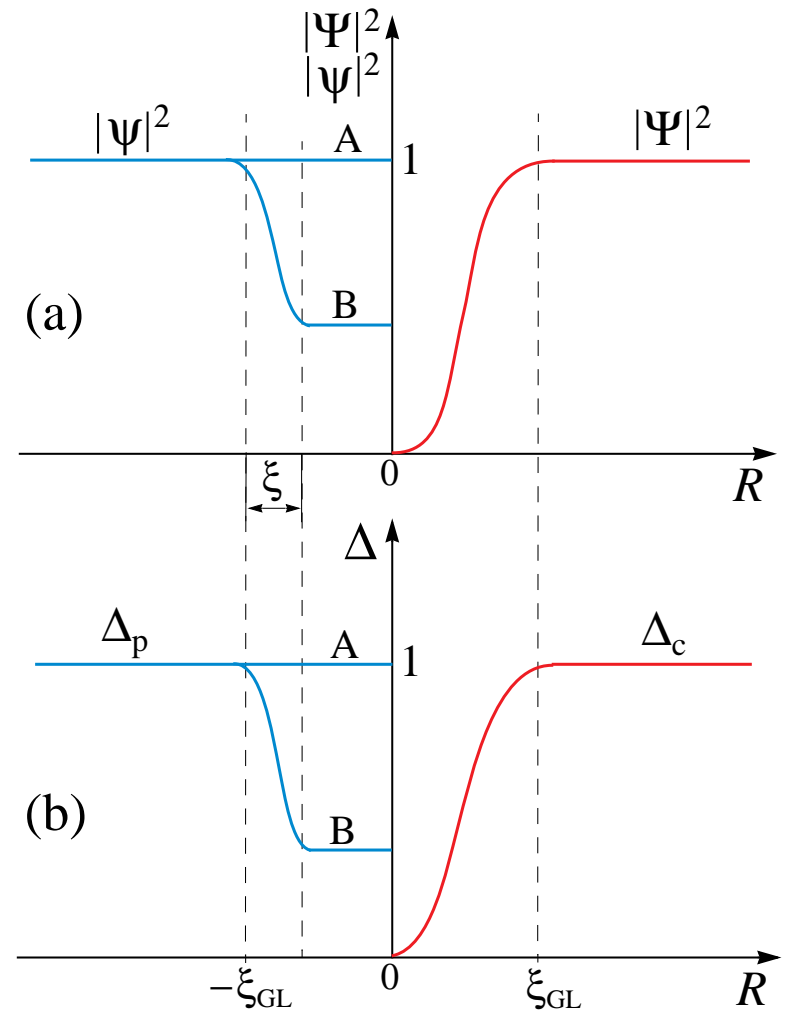

FIG. 8. Spatial variations of (a) the order parameter $|\Psi|^{2}$ and the averaged wavefunction $|\psi|^{2}$, and (b) the energy gaps $\Delta_{p}$ and $\Delta_{c}$ inside and outside an isolated vortex in an infinite unconventional superconductor with a Russian-doll-like condensate. $R$ is the distance from the center of the vortex. $\xi_{G L}$ is the coherence length, and $\xi$ is the Cooper-pair size. For simplicity, all the functions are normalized at $R=\infty$ to 1 . Since the pairing wavefunction $\psi$ is not global, $\psi$ in plot (a) is average. The letters $\mathrm{A}$ and $\mathrm{B}$ indicate a different behaviour of $|\psi|^{2}$ and $\Delta_{p}$.

pairing temperature $T_{p}$ is sufficiently high and there are structural transitions below $T_{p}, \xi$ will vary with temperature.

Let us consider the variations of the order parameter and the Cooper-pair wavefunction into the vortex cores appearing in the mixed state. The spatial variations of the order parameter $|\Psi|^{2}$ and the averaged wavefunction $|\psi|^{2}$ into a vortex core are sketched in Fig. 8(a). Inside a vortex core, the order parameter always goes to zero, while $|\psi|^{2}$ must not. Depending on the coupling strength of the Cooper pairs, there are two possible outcomes for the behaviour of $|\psi|^{2}$ into a vortex core, shown by the letters A and B in Fig. 8(a). The spatial variation of the averaged $|\psi|^{2}$ wavefunction, if such occurs, is of the order of $\xi$ in the clean limit. The coherence energy gap $\Delta_{c}(\propto|\Psi|)$ will be always zero inside a vortex core, as depicted in Fig. 8(b). In contrast, the pairing energy gap $\Delta_{p}$ will not be zero into a vortex core, and even, may remain unchanged [the case $\mathrm{A}$ in Fig. 8(b)]. In the latter case, the size of the Cooper pairs cannot be

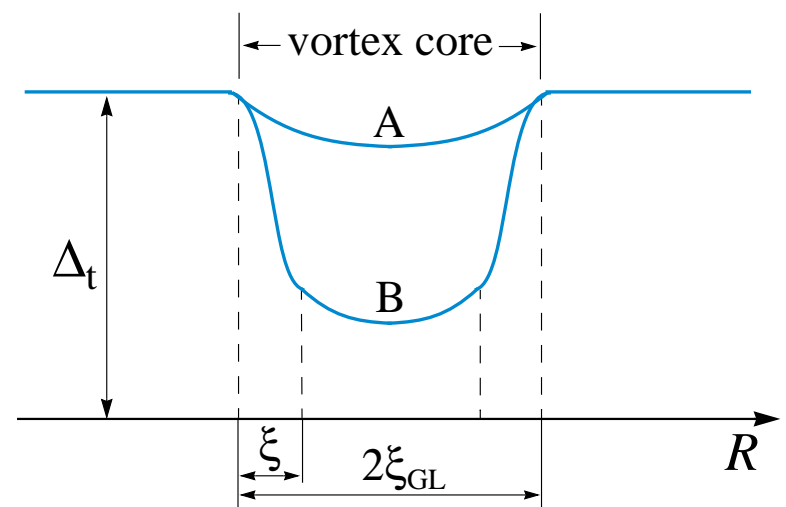

FIG. 9. Spatial variations of the total energy gap $\Delta_{t}=\left(\Delta_{p}^{2}+\Delta_{c}^{2}\right)^{1 / 2}$ inside and outside an isolated vortex in an infinite unconventional superconductor with a Russian-doll-like condensate $\left(\Delta_{c}<\Delta_{p}\right)$. The line $R$ passes through the center of the vortex core. $\xi_{G L}$ is the coherence length, and $\xi$ is the size of Cooper pairs. The letters A and $\mathrm{B}$ correspond to the cases shown in Fig. 8(b).

determined from the tunnelling data. In comparison with the case of two-band superconductors (Fig. 5), $|\psi|^{2}$ and $\Delta_{p}$ will never be zero inside a vortex: the presence of the long-range phase coherence in a superconductor with a Russian-doll-like condensate in the absence of the Cooper pairs has no sense.

In superconductors in which the superconducting condensate has a structure similar to that of a Russian doll, the total energy gap below $T_{c}$ is $\Delta_{t}=\sqrt{\Delta_{p}^{2}+\Delta_{c}^{2}}$ [17]. Therefore, tunnelling measurements performed in vortex cores of such unconventional superconductors should theoretically show the spatial variation of the total energy gap, as illustrated in Fig. 9. Since always $\Delta_{c}<\Delta_{p}$ [17], the spatial variations of $\Delta_{t}$ should be more pronounced within $\xi$ from the walls of a vortex (the cases B and C in Fig. 9). From the data, one can estimate not only the value of the coherence length but also the value of $\xi$.

In real superconductors, however, the situation can be different. Let us consider an example: assume that in a superconductor with a Russian-doll-like condensate, magnetic fluctuations mediate the long-range phase coherence. Tunnelling measurements probe the local density of states of quasiparticle excitations present on the surface. It is known that, in magnetic materials, the spectrum of magnetic excitations on the surface differs from that in the bulk [4]. Depending on the quality of the surface, spin fluctuations into the topmost layer may not be able to mediate the long-range phase coherence. Therefore, the Cooper pairs on the surface will remain uncondensed. As a consequence, in tunnelling measurements performed in this superconductor, the pairing energy gap $\Delta_{p}$ will be predominant in the spectra, and its spatial variations into a vortex core will be similar to those shown in Fig. 6(b). 


\section{B. Condensate with two types of Cooper pairs}

The second type of unconventional superconductors which we are going to discuss now is the superconductors with a condensate consisting of two different types of Cooper pairs, which are independent of one another. These two types of Cooper pairs are formed due to different bosonic excitations. The Cooper pairs of one type, for example, are formed due to phonons, while spin fluctuations, for instance, are responsible for the formation of Cooper pairs of the second type. The long-range phase coherence is mediated by the overlap of the wavefunctions of the Cooper pairs of one of these types, meaning that this type of the Cooper pairs appears at $T_{c}$. Assume that the Cooper pairs of the other type are formed above the critical temperature, $T_{p}>T_{c}$. In these unconventional superconductors, the symmetries of the two types of the Cooper-pair wavefunctions can be different, as in the case of superconductors with a Russian-doll-like condensate.

Let us consider two possible cases of the spatial distribution of the Cooper pairs. If the two types of the Cooper pairs "penetrate" one another on a nanoscale, this situation is basically equivalent to the case of halfconventional superconductors, discussed above. Therefore, tunnelling spectroscopy data obtained along a line passing through the center of a vortex core in such superconductors should be similar to those obtained in halfconventional superconductors. As a consequence, Figures 4-6 are in principle applicable to this case, and the spatial variations of the two gaps are sketched in Fig. 6. Depending on the quality of the surface, one can detect both gaps simultaneously or one at a time. From these data, one can in principle estimate the values of $\xi_{G L}$ and/or $\xi$.

If the two types of the Cooper pairs do not mix on a nanoscale, the superconducting phase will then have the "shape" of the Swiss cheese with the incoherent Cooper pairs of the other type inside the "cavities" (in layered superconductors, "pancakes"). On the surface, this structure will look like the coexistence of two types of alternating patches. In the mixed state, the vortices will penetrate the superconductor in the weakest spots, i.e. in spots where the minimum free energy is needed to break the superconducting phase. In this case, the vortex lattice may be not regular. On the other hand, due to a strong interaction between vortices, the vortex lattice can become regular, and the "weakest superconducting phase" will follow the vortices. In any case, by performing tunnelling measurements along a line passing through the center of a vortex core in such unconventional superconductors, one can get information only about one energy gap and, respectively, can estimate either $\xi_{G L}$ or $\xi_{2}$ (the size of the incoherent Cooper pairs). The spatial variations of the two energy gaps will be similar to those sketched in Fig. 6.

\section{VORTEX-CORE TUNNELLING DATA OBTAINED IN REAL SUPERCONDUCTORS}

In the literature, one can find only a few sets of tunnelling spectroscopy data obtained in the mixed state of half-conventional and unconventional superconductors [5-14]. Here we discuss these data, expecting to estimate the values of the coherence length and the size of the Cooper pairs in respective superconductors. All superconductors which will be considered in this section are obviously type-II superconductors. We start with halfconventional superconductors. All the data presented in this section are acquired in superconductor-insulatornormal metal junctions.

\section{A. Half-conventional superconductors}

The recently discovered magnesium diboride $\left(\mathrm{MgB}_{2}\right)$ superconductor is a typical half-conventional superconductor $[4,17]$. Ironically enough, it is probably the most studied half-conventional superconductor.

In $\mathrm{MgB}_{2}$, superconductivity occurs in the boron layers. Band-structure calculations of $\mathrm{MgB}_{2}$ show that there are at least two types of bands at the Fermi surface. The first one is a narrow band, built up of boron $\sigma$ orbitals, whilst the second one is a broader band with a smaller effective mass, built up mainly of $\pi$ boron orbitals.

The presence of two energy gaps in $\mathrm{MgB}_{2}$ is a well documented experimentally. The larger energy gap $\Delta_{\sigma}$ occurs in the $\sigma$-orbital band, the smaller gap $\Delta_{\pi}$ in the $\pi$-orbital band. The gap ratio $2 \Delta /\left(k_{B} T_{c}\right)$ for $\Delta_{\sigma}$ is about 4.5. For $\Delta_{\pi}$, this ratio is around 1.7, so that, $\Delta_{\sigma} / \Delta_{\pi} \simeq$ 2.7. Both energy gaps have an s-wave symmetry. The larger gap is highly anisotropic, while the smaller one is either isotropic or slightly anisotropic. The induced character of $\Delta_{\pi}$ manifests itself in its temperature dependence: if $\Delta_{\sigma}$ follows the temperature dependence derived in the framework of the BCS theory, the temperature dependence of $\Delta_{\pi}$ lies below the BCS dependence at $T \rightarrow T_{c}$.

Tunnelling spectroscopy measurements have been performed in $\mathrm{MgB}_{2}$ in the mixed state along a line passing through the center of a vortex core [13]. Since the measurements have been conducted along the $c$-axis, they have been tuned outside of the vortex core to $\Delta_{\pi}$ which is the coherence gap in $\mathrm{MgB}_{2}$. As the authors admit, "the spectra in the center of a vortex are absolutely flat, ..." [13]. This result is in good agreement with Fig. 6(a). The inner structure of a vortex would be complex if the measurements would be initially tuned to $\Delta_{\sigma}$. The coherence length $\xi_{G L}$ estimated from the size of vortex cores is about $500 \AA$ [13].

The behaviour of $\Delta_{\sigma}$ in the presence of magnetic field is also documented in the literature. Figure 10 shows a set of tunnelling conductances recorded perpendicular 


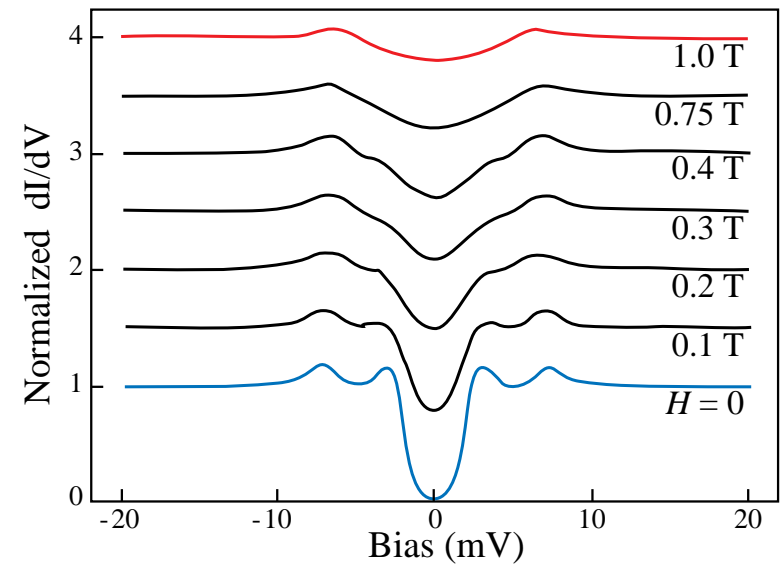

FIG. 10. Tunnelling conductances obtained in $\mathrm{MgB}_{2}$ at 2 $\mathrm{K}$ and different magnetic fields [14]. The spectra are recorded between the vortices. They are offset vertically for clarity. The critical temperature of the sample is $37.7 \mathrm{~K}$.

to the $c$-axis in applied magnetic fields up to $1 \mathrm{~T}$. The conductances are obtained between vortices, not inside of a vortex. In Fig. 10, the conductance at zero field has a double-gap structure reflecting the presence of both $\Delta_{\pi}$ and $\Delta_{\sigma}$. As the field is increased, the inner peaks associated with $\Delta_{\pi}$ are rapidly suppressed, and the zerobias conductance increases. The outer peaks reflecting $\Delta_{\sigma}$ are affected by the field much less than those from $\Delta_{\pi}$. In the absence of the field, $\Delta_{\sigma} \simeq 7.2 \mathrm{meV}$, and at $H$ $=1 \mathrm{~T} \Delta_{\sigma} \simeq 6.6 \mathrm{meV}$. If these spectra were taken along a line passing through the center of a vortex, they would correspond to the case B in Fig. 6(b).

The second half-conventional superconductor which was also intensively studied in the mixed state by tunnelling spectroscopy is $2 \mathrm{H}-\mathrm{NbSe}_{2}$ [5-7]. $2 \mathrm{H}-\mathrm{NbSe}_{2}$ is a "clean" layered superconductor with the presence of charge-density waves (thus, it is low-dimensional). Below $T_{c}, 2 \mathrm{H}-\mathrm{NbSe}_{2}$ has two energy gaps, and the ratio $\Delta_{L} / \Delta_{S}$ is about $3[23,24]$. As a consequence, $\xi_{G L} / \xi \sim 3$ too. The conductances obtained in the center of a vortex exhibit a a peak at zero bias which will be discussed below. The coherence length $\xi_{G L}$ estimated from the size of vortex cores is about $175 \AA$.

\section{B. Unconventional superconductors}

In this subsection, we shall discuss tunnelling spectroscopy data obtained in three unconventional superconductors. We start with the data measured in $\mathrm{LuNi}_{2} \mathrm{~B}_{2} \mathrm{C}$ [9].

The nickel borocarbide class of superconductors has the general formula $R \mathrm{Ni}_{2} \mathrm{~B}_{2} \mathrm{C}$, where $R$ is a rare earth which is either magnetic (Tm, Er, Ho, or Dy) or nonmagnetic ( $\mathrm{Lu}$ and $\mathrm{Y})$. The Ni borocarbides have a layeredtetragonal structure alternating $R \mathrm{C}$ sheets and $\mathrm{Ni}_{2} \mathrm{~B}_{2}$ layers. Transition temperatures in these quaternary in-

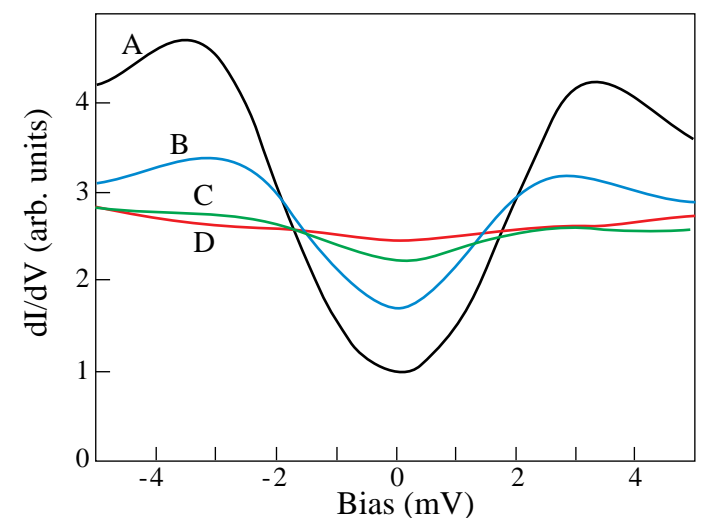

FIG. 11. Tunnelling conductances obtained in $\mathrm{LuNi}_{2} \mathrm{~B}_{2} \mathrm{C}$ at $4.2 \mathrm{~K}$ and $0.375 \mathrm{~T}$ [9]. The spectra are recorded at 470 $\AA$ (curve A), $130 \AA$ (B) and $20 \AA$ (C) from the center of a vortex. The curve $\mathrm{D}$ is measured in the center of the vortex. The critical temperature of the sample is $15.8 \mathrm{~K}$.

termetallic compounds can be as high as $17 \mathrm{~K}$. In the case when $R=\mathrm{Pr}, \mathrm{Nd}, \mathrm{Sm}, \mathrm{Gd}$ or $\mathrm{Tb}$ in $R \mathrm{Ni}_{2} \mathrm{~B}_{2} \mathrm{C}$, the $\mathrm{Ni}$ borocarbides are not superconducting at low temperatures but antiferromagnetic. In the Ni borocarbides with a magnetic rare earth, superconductivity coexists at low temperatures with a long-range antiferromagnetic order.

Many different types of measurements carried out in the $\mathrm{Ni}$ borocarbides show that the gap ratio $2 \Delta /\left(k_{B} T_{c}\right)$ is between 3.2 and 5.3 [17]. What concerns the shape of the energy gap, there is complete disagreement in the literature. In photoemission and microwave measurements, the energy gap in some $\mathrm{Ni}$ borocarbides was found to be an s-wave but highly anisotropic [17]. On the other hand, in specific-heat, thermal-conductivity and Ramanscattering measurements carried out in the Ni borocarbides with $R=\mathrm{Y}$ and $\mathrm{Lu}$, the energy gap was found to be a highly anisotropic gap, most likely with nodes. Furthermore, in other thermal-conductivity measurements, the gap appears to have point nodes along the [100] and [010] directions, thus along the $a$ and $b$ axes. Recent tunnelling measurements performed in the antiferromagnetic $\mathrm{TmNi}_{2} \mathrm{~B}_{2} \mathrm{C}$ unambiguously show that this $\mathrm{Ni}$ borocarbide is a fully gapped s-wave superconductor with a gap being slightly anisotropic. To reconcile all these data, one should assume that different measurements probe different energy gaps, either $\Delta_{p}$ or $\Delta_{c}$.

Figure 11 depicts tunnelling conductances obtained in the mixed state in the $\mathrm{LuNi}_{2} \mathrm{~B}_{2} \mathrm{C}$ borocarbide at various distances from the center of a vortex. All the conductance in Fig. 11, including that recorded in the center of a vortex, have a gap structure. However, the humps in the conductances obtained inside the vortex are not situated symmetrically relatively zero. The bias positions of the left-hand humps in the spectra are $V_{l}(470 \AA) \simeq$ $3.4 \mathrm{mV}, V_{l}(130 \AA) \simeq 3.1 \mathrm{mV}, V_{l}(20 \AA) \simeq 2.5 \mathrm{mV}$, and $V_{l}(0 \AA) \simeq 1.8 \mathrm{mV}$. The bias positions of the right-hand humps in the spectra are $V_{r}(470 \AA) \simeq 3.4 \mathrm{mV}, V_{r}(130 \AA)$ 


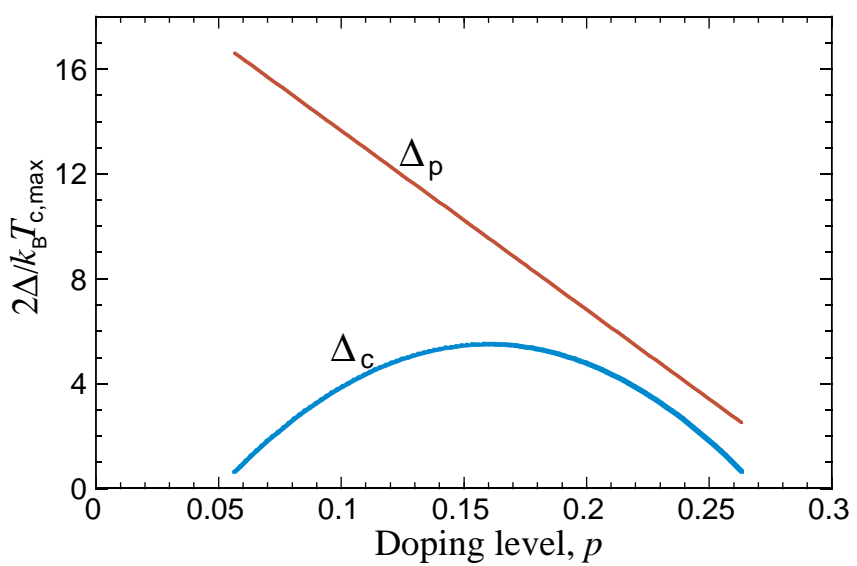

FIG. 12. The phase diagram of hole-doped cuprates $[25,26,4,17] . \Delta_{c}$ is the phase-coherence energy gap, and $\Delta_{p}$ is the pairing energy gap.

$\simeq 2.9 \mathrm{mV}, V_{r}(20 \AA) \simeq 3.0 \mathrm{mV}$, and $V_{r}(0 \AA) \simeq 3.1 \mathrm{mV}$. The bias positions of the humps are determined with an error of about $\pm 0.1 \mathrm{mV}$. Unfortunately, from these data, it is impossible to determine what type of unconventional superconductors $\mathrm{LuNi}_{2} \mathrm{~B}_{2} \mathrm{C}$ belongs to (see the previous section). The evolution of the hump position on the lefthand side of the conductances is in agreement with the case A in Fig. 9. At the same time, the evolution of the hump position on the right-hand side of the conductances is in agreement with the case B in Fig. 6(b). Indeed, the gap ratio $2 \Delta(470 \AA) / k_{B} T_{c} \simeq 4.9$ indicates that the spectra in Fig. 11 reflect either a $\Delta_{p}\left(\Delta_{L}\right)$ or a $\Delta_{t}$ gap (see above). The radius of the vortex cores in $\mathrm{LuNi}_{2} \mathrm{~B}_{2} \mathrm{C}$ is about $200 \pm 10 \AA$. Then, $\xi_{G L} \approx 200 \AA$. If we rely on the bias position of the right-hand humps, then one can estimate the size of the Cooper pairs: $\xi \sim 200-130=70$ $\AA$. If this is the case, then $\Delta_{L} / \Delta_{S} \propto \xi_{G L} / \xi \sim 3$. Unfortunately, the set of the data presented in Fig. 11 is too limited for more definitive evaluation of the size of the Cooper pairs.

Let us now discuss tunnelling data obtained in the mixed state of two cuprates: $\mathrm{YBa}_{2} \mathrm{Cu}_{3} \mathrm{O}_{7-\delta}(\mathrm{YBCO})$ and $\mathrm{Bi}_{2} \mathrm{Sr}_{2} \mathrm{CaCu}_{2} \mathrm{O}_{8}$ (Bi2212). The superconducting phase in cuprates appears when they are slightly doped. The critical temperature of cuprates can be tuned by varying the doping level. Hole-doped cuprates have two energy gaps related to the superconducting phase, $\Delta_{p}$ and $\Delta_{c}$, shown in Fig. 12. The electron-doped cuprates have a similar phase diagram $[27,4]$.

Figure 13 shows two averaged tunnelling conductances recorded in the mixed state of Bi2212. The conductance obtained in the center of a vortex has two features: an apparent enlargement of the gap magnitude and the presence of a subgap. The subgap corresponds to the gap inside the cortex cores, the magnitude of which is proportional to the magnitude of the gap recorded outside of the vortices with the coefficient of about 0.3 [12]. Thus, the subgap in the lower conductance in Fig. 13 is either

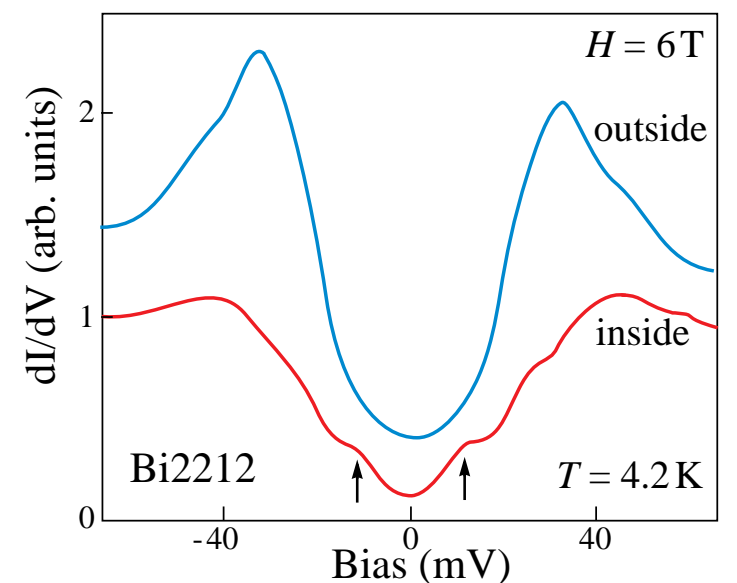

FIG. 13. Averaged spectra recorded in the center of a vortex and outside the vortex in overdoped $\operatorname{Bi} 2212\left(T_{c}=87.4 \mathrm{~K}\right)$ at $4.2 \mathrm{~K}$ and $6 \mathrm{~T}$ [12]. The arrows indicate a subgap structure. The upper curve is offset vertically for clarity.

$\Delta_{p}$ [the case B in Fig. 6(b)] or $\Delta_{t}$ [the case B in Fig. 9] with a reduced magnitude. From the data presented in [12], it is very difficult to distinguish between these two possible cases: seemingly, the value of the subgap remain constant near to the center of the vortex (see Fig. 2(a) in [12]). So, it is more likely that the subgap in the lower conductance in Fig. 13 corresponds to $\Delta_{p}$. The radius of the vortex core in slightly overdoped Bi2212 is about $22 \pm 3 \AA$ [11]. Because of the small size of the vortices in Bi2212 and a weak manifestation of the subgap in the spectra [12], it is practically impossible to estimate the size of the Cooper pairs in Bi2212 from the data. The only way to estimate $\xi$ is from the ratio $\xi / \xi_{G L} \sim \Delta_{c} / \Delta_{p}$. Then, by using Fig. 12 one can obtain that in slightly overdoped Bi2212, $\xi \sim 22 \times 20 / 32 \AA \simeq 14 \AA$.

The increase of the magnitude of the gap in Fig. 13 is apparent. The quasiparticle peaks in tunnelling conductances recorded below $T_{c}$ in cuprates appear on top of a contribution from a normal-state pseudogap $[28,29,4]$. The disappearance of the peaks discloses the humps from the pseudogap. Since the magnitude of the pseudogap is larger than the magnitudes of $\Delta_{p}$ and $\Delta_{c}$, this creates an effect of an increase of the magnitude of the gap inside the vortex cores. This can be demonstrated by using the data from [10]: in Fig. 14, one can see that the conductance recorded in the center of a vortex is very similar to the conductance obtained above $T_{c}$. The gap structure in both these curves is caused by a normal-state pseudogap which is always present "beneath" the superconducting gap(s). The same effect of an apparent increase of the magnitude of the gap inside the vortex cores also occurs in YBCO, as illustrated in Fig. 15.

The subgap structures in conductances recorded in the center of a vortex are more pronounced in YBCO than those in Bi2212, as shown in Fig. 15. The problem, however, is that this subgap is caused not only by the 


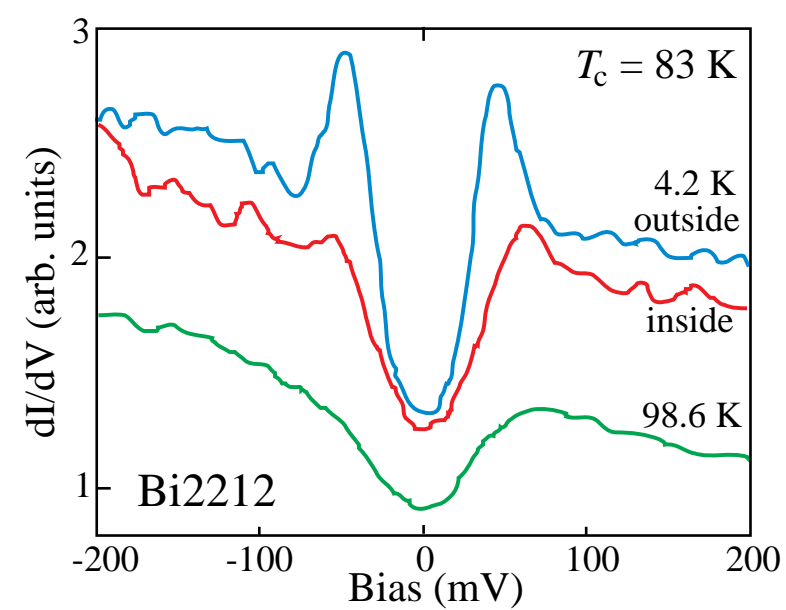

FIG. 14. Tunnelling conductances obtained in underdoped Bi2212 with $T_{c}=83 \mathrm{~K}[10]$. The lower conductance is taken in zero magnetic field at $98.6 \mathrm{~K}$. The other curves are measured at $4.2 \mathrm{~K}$ and $6 \mathrm{~T}$. The upper curve is recorded between vortices, and the middle curve is acquired in the center of a vortex. For clarity the $4.2 \mathrm{~K}$ curves are offset vertically.

superconducting gap with a reduced magnitude, as that in Bi2212, but also by the gap on chains. In the crystal structure, the unit cell of $\mathrm{YBCO}$ has four $\mathrm{CuO}$ chains which are parallel to the $b$ crystal axis. YBCO is the only superconducting cuprate having one-dimensional $\mathrm{CuO}$ chains. In $\mathrm{YBCO}$, the $\mathrm{CuO}$ chains become superconducting due to the proximity effect [17]. The value of the superconducting energy gap on the chains in optimally doped YBCO is about $6 \mathrm{meV}$ [4]. In Fig. 15, one can see that even the conductance obtained without an applied magnetic field has a subgap structure caused by the superconducting energy gap on the chains. In the vortex cores, the bulk superconducting gap with a reduced magnitude appears in tunnelling spectra "on top" of the superconducting gap on $\mathrm{CuO}$ chains. Because of this, it is impossible to estimate of the Cooper-pair size from the data presented in [8]: the weak subgap in conductances recorded outside a vortex smoothly transforms to a more-pronounced subgap in conductances acquired in the vortex core, having a very similar magnitude (see Fig. 3(b) in [8]). In YBCO, the size of the vortex is at least a factor of 2 larger than that in Bi2212 [10], thus, it is slightly larger than $40 \AA$. However, the vortex size in the bulk of YBCO, determined by $\mu \mathrm{SR}$ at $6 \mathrm{~T}$, is about $20 \AA$ [3]. It is also worth to mention that, in Fig. 15, the magnitude of the energy gap in the conductance recorded without an applied magnetic field corresponds to $\Delta_{c}$ in Fig. 12, and not to $\Delta_{p}$, as those in Figs. 13 and 14 in Bi2212.

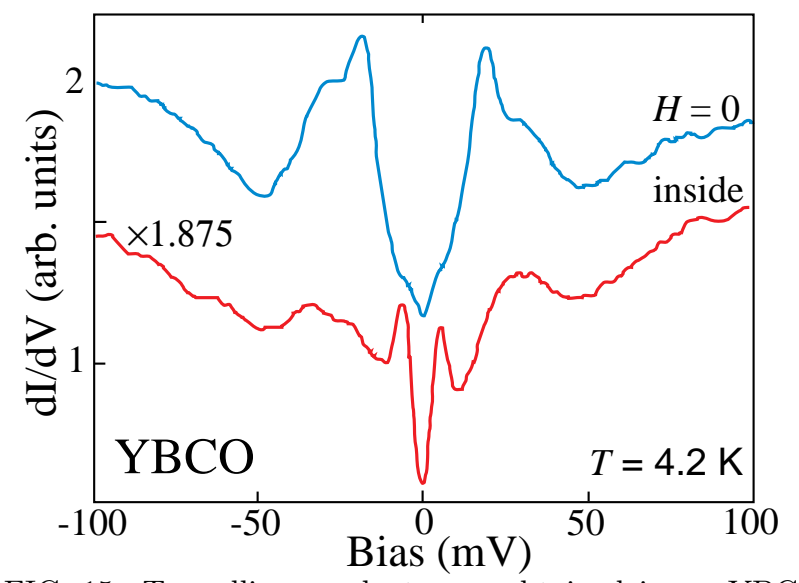

FIG. 15. Tunnelling conductances obtained in an YBCO single crystal with $T_{c}=91 \mathrm{~K}$ at $4.2 \mathrm{~K}$ [8]. The upper curve was measured in zero magnetic field. The lower curve is the averaged spectrum of conductances obtained at the center of a vortex core along a $5 \mathrm{~nm}$ path and in $H=6 \mathrm{~T}$ applied along the $c$ axis. The lower curve was magnified by a factor of 1.875. The upper curve is offset vertically for clarity.

\section{Zero-bias conductance peak}

Tunnelling conductances obtained in the center of a vortex in $2 \mathrm{H}-\mathrm{NbSe}_{2}$ exhibit a peak at zero bias [5-7] shown in Fig. 16. It is not the purpose of this paper to discuss the origin of this peak. However, it is worth to spent some time on this issue. Generally speaking, the zero-bias conductance peak is a manifestation of some bound states inside the vortex cores. The question is what kind of bound states are they?

As shown in [4], solitonic states present in a system will manifest themselves in tunnelling conductances through the appearance of a peak at zero bias. In superconductorinsulator-normal metal junctions, the shape of this zerobias conductance peak will be proportional to a $\operatorname{sech}^{2}$ function as follows

$$
\frac{d I(V)}{d V}=A \times \operatorname{sech}^{2}\left(V / V_{0}\right),
$$

where $V$ is the applied bias, and $A$ and $V_{0}$ are the constants. The corresponding $I(V)$ characteristic is

$$
I(V)=A V_{0} \times \tanh \left(V / V_{0}\right) .
$$

It is interesting that the zero-bias conductance peak recorded in the center of vortex cores in $2 \mathrm{H}-\mathrm{NbSe}_{2}$ also has the shape of a $\operatorname{sech}^{2}$ function, as depicted in Fig. 16. Superconductivity in $2 \mathrm{H}-\mathrm{NbSe}_{2}$ coexist with chargedensity waves which are quasi-one dimensional. Solitons require the presence of quasi-one-dimensionality in the system. So, it is possible that the zero-bias peak in conductances recorded in the center of vortex cores in $2 \mathrm{H}$ $\mathrm{NbSe}_{2}$ is a manifestation of solitonic states in $2 \mathrm{H}-\mathrm{NbSe}_{2}$ (which may be paired outside the vortex cores). 


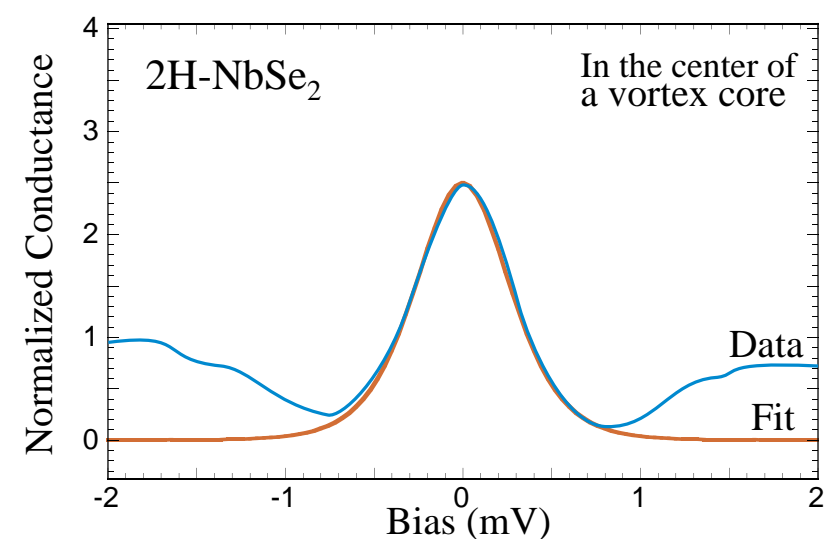

FIG. 16. Tunnelling conductance recorded in the center of a vortex in $2 \mathrm{H}-\mathrm{NbSe}_{2}$ and the $A \times \operatorname{sech}^{2}\left(V / V_{0}\right)$ fit. The data are taken from Fig. 2 in [6]. In the fit, $A=2.5$ and $V_{0}=1 / 2.8$ $\mathrm{mV}$.

A peak at zero bias also appear in conductances recorded in non-superconducting and superconducting compounds without an applied magnetic field. In the charge-density-wave conductor $\mathrm{NbSe}_{3}$, a zero-bias conductance peak has the shape of a $\operatorname{sech}^{2}$ function too [30]. In superconducting hole-doped [4] and electrondoped $[27,4]$ cuprates, a peak at zero bias occurring in conductances recorded usually at $45^{\circ}$ relative to the inplane crystal axes has also the shape of a $\operatorname{sech}^{2}$ function.

\section{DISCUSSION}

It is necessary to note that the determination of the values of the coherence length and the size of the Cooper pairs in unconventional superconductors by using tunnelling spectroscopy is not a straight forward procedure. In unconventional superconductors, the values of the coherence length and the size of the Cooper pairs are usually small. As a consequence, the values of $\xi_{G L}$ and, in particular, $\xi$ estimated from the experiment will have a large error. In addition to this, there are at least three other aspects affecting the results. First, depending on the crystal structure, the shape of vortex cores can be not round: in $2 \mathrm{H}-\mathrm{NbSe}_{2}$, the vortex cores have the shape of a six-leg star [6]. Second, the size of vortex cores varies: it depends on the distance between the vortices, i.e. finally, on $H$. Third, the vortices slightly expand near the surface. The size of a vortex can increase by up to $30 \%$ at the sample surface in comparison with that in the bulk [3]. A discussion concerning the structure and the size of vortex cores can be found elsewhere $[31,3]$.

In the framework of the Ginzburg-Landau theory, the value of the coherence length in type-II superconductors can be determined from $H_{c 2}$ as follows

$$
\xi_{G L}^{2}=\frac{\Phi_{0}}{2 \pi H_{c 2}},
$$

where

$$
\Phi_{0} \equiv \frac{h}{2 e}=2.0679 \times 10^{-15} \quad \mathrm{~T} \mathrm{~m}^{2} \text { (or Weber) }
$$

is the magnetic flux quantum. In layered unconventional superconductors, the critical magnetic field $H_{c 2}$, as well as $\xi_{G L}$, is different in different directions-parallel and perpendicular to the layers. For example, the upper critical field applied perpendicular to the layers, $H_{c 2, \perp}$, is determined by vortices whose screening currents flow parallel to the planes. For layered superconductors, the Ginzburg-Landau expression takes the following form

$$
H_{c 2, \perp}=\frac{\Phi_{0}}{2 \pi \xi_{G L, a b}^{2}},
$$

where the letters "ab" indicate that the direction of the screening currents is in the ab-plane. The value of the coherence length perpendicular to the planes, $\xi_{G L, c}$ can be determined from the ratio

$$
\frac{H_{c 2, \|}}{H_{c 2, \perp}}=\frac{\xi_{G L, a b}}{\xi_{G L, c}}
$$

where $H_{c 2, \|}$ is the upper critical field applied parallel to the layers.

In reality, however, the values of $\xi_{G L, a b}$ and $\xi_{G L, c}$, determined in unconventional superconductors through $H_{c 2, \|}$ and $H_{c 2, \perp}$ are not the in-plane and out-of-plane coherence lengths - they correspond to the size of the Cooper pairs in two directions, i.e. to $\xi_{a b}$ and $\xi_{c}$ [4]. As an example, let us consider the doping dependence of the in-plane coherence length and Cooper-pair size in $\mathrm{La}_{2-x} \mathrm{Sr}_{x} \mathrm{CuO}_{4}$ (LSCO). The lower curve in Fig. 17 is the in-plane characteristic length in LSCO determined from the $H_{c 2, \perp}(p)$ dependence. It is easy to check that the dependence $1 / \xi_{a b}(p)$ from Fig. 17 does not mimic the $\Delta_{c}(p)$ dependence in Fig. 12. Instead, it is similar to the $\Delta_{p}(p)$ dependence in Fig. 12. In fact, the upper curve in Fig. 17 represents $\xi_{G L}(p)$ in LSCO which will be discussed in a moment. Let us first consider other examples: in $\mathrm{MgB}_{2}$, the value of the coherence length determined from the size of vortex cores is about $500 \AA$ (see above), while determined from $H_{c 2}$ is about $100 \AA$ [13]. In $2 \mathrm{H}-\mathrm{NbSe}_{2}, \xi_{G L} \sim 175 \AA$, while $\xi \sim$ $77 \AA$, respectively [6]. In $\mathrm{LuNi}_{2} \mathrm{~B}_{2} \mathrm{C}, \xi_{G L} \sim 200 \AA$, while $\xi \sim 75 \AA$, respectively [9]. In slightly overdoped Bi2212, $\xi_{G L} \sim 22 \AA$ (see above), while $\xi \sim 14 \AA$ determined from $H_{c 2}=\Phi_{0} /\left(2 \pi \xi^{2}\right)$. All these values are low-temperature values. So, experimentally, the value of the coherence length estimated from the vortex-core size is always larger than that obtained from the GinzburgLandau expression $H_{c 2}=\Phi_{0} /\left(2 \pi \xi_{G L}^{2}\right)$. In unconventional superconductors, the $H_{c 2}$ method provides the values of the Cooper-pair size.

In unconventional superconductors, it is possible to estimate the values of $\xi$ and $\xi_{G L}$ independently. For example, the Cooper-pair size at low temperature can be 


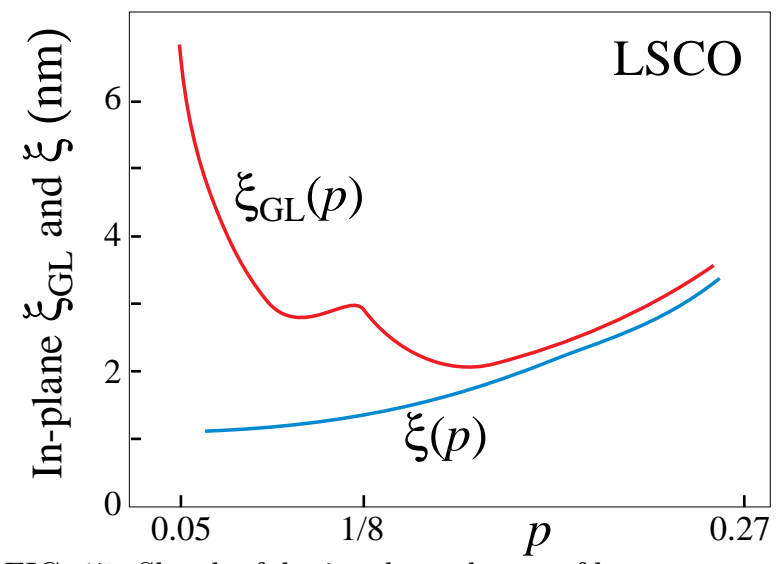

FIG. 17. Sketch of doping dependences of low-temperature in-plane $\xi_{G L}(0)$ and $\xi(0)$ in LSCO. The Cooper-pair size is obtained from $H_{c 2}=\Phi_{0} /\left(2 \pi \xi^{2}\right)$ [32].

obtained from Eq. (1). To estimate the size of vortex cores is more complicated - such an approach is described elsewhere [32]. Figure 17 depicts the doping dependence of $\xi_{G L, a b}$ in LSCO, derived by using the doping dependences of other superconducting characteristics, such as the critical current density, the collective pinning energy, the superfluid density and the condensation energy. Indeed, the $1 / \xi_{G L, a b}(p)$ dependence from Fig. 17 is similar to the doping dependence $\Delta_{c}(p)$ in Fig. 12. At $p \simeq 1 / 8$, $\xi_{G L, a b}(p)$ has a kink related to the so-called $\frac{1}{8}$ anomaly inherent exclusively to LSCO. This fact indicates that the disappearance of superconductivity in LSCO at $p \simeq 1 / 8$ is due to the absence of the long-phase coherence, not the Cooper pairs.

\section{CONCLUSIONS}

The main purpose of the paper was to discuss a possibility of the determination of the values of the coherence length and the Cooper-pair size in unconventional superconductors by using tunnelling spectroscopy. In unconventional superconductors, the inner structure of a vortex core has a complex structure determined by the order parameter of the superconducting state and by the pairing wavefunction of the Cooper pairs. In clean superconductors, the spatial variations of the order parameter and the pairing wavefunction occur over the distances of the order of the coherence length and the Cooper-pair size, respectively. Therefore, by performing tunnelling spectroscopy along a line passing through a vortex core, one is able, in principle, to estimate the values of the coherent length and the Cooper-pair size. In the paper, common patterns of the structure of the vortex cores have been obtained for two-band (half-conventional) superconductors and unconventional superconductors. At the moment, a detail comparison tunnelling spectroscopy data recorded in half-conventional and unconventional superconductors and the derived patterns is not possible because the set of tunnelling spectroscopy data available in the literature is limited. In the future, both the spatial and energy resolutions of tunnelling spectroscopy measurements need to be improved since the size of the vortex cores in unconventional superconductors is very small.

\section{Acknowledgements}

I thank J. E. Sonier for comments on the manuscript.

[1] V. L. Ginzburg and L. D. Landau, Zh. Eksper. Teor. Fiz. 20, 1064 (1950).

[2] A. A. Abrikosov, Sov. Phys. JETP 5, 1174 (1957).

[3] J. E. Sonier, J. H. Brewer, and R. F. Kiefl, Rev. Mod. Phys. 72, 769 (2000).

[4] A. Mourachkine, High-Temperature Superconductivity in Cuprates: The Nonlinear Mechanism and Tunnelling Measurements, (Kluwer Academic, Dordrecht, 2002), Chapter 12, pp. 251, 67, 295, 145, 156, 138, 283, 292, 234.

[5] H. F. Hess, R. B. Robinson, R. C. Dynes, J. M. Valles, Jr., and J. V. Waszczak, Phys. Rev. Lett. 62, 214 (1989).

[6] H. F. Hess, R. B. Robinson, and J. V. Waszczak, Phys. Rev. Lett. 64, 2711 (1990).

[7] Ch. Renner, A. Kent, Ph. Niedermann, O. Fisher, and F. Levy, Phys. Rev. Lett. 67, 1650 (1991).

[8] I. Maggio-Aprili, Ch. Renner, A. Erb, E. Walker, and O. Fisher, Phys. Rev. Lett. 75, 2754 (1995).

[9] Y. De Wilde, M. Iavarone, U. Welp, V. Metlushko, A. E. Koshelev, I. Aranson, G. W. Grabtree, and P. C. Canfield, Phys. Rev. Lett. 78, 4273 (1997).

[10] Ch. Renner, B. Revaz, K. Kadowaki, I. Maggio-Aprile, and O. Fischer, Phys. Rev. Lett. 80, 3606 (1998).

[11] S. H. Pan, E. W. Hudson, A. K. Gupta, K.-W. Ng, H. Eisaki, S. Uchida, and J. C. Davis, Phys. Rev. Lett. 85, 1536 (2000).

[12] B. W. Hoogenboom, K. Kadowaki, B. Revaz, M. Li, Ch. Renner, and O. Fisher, Phys. Rev. Lett. 87, 267001 (2001).

[13] M. R. Eskildsen, M. Kugler, S. Tanaka, J. Jun, S. M. Kazakov, J. Karpinski, and O. Fisher, Phys. Rev. Lett. 89, 187003 (2002).

[14] M. R. Eskildsen, N. Jenkins, G. Levy, M. Kugler, O. Fisher, J. Jun, S. M. Kazakov, and J. Karpinski,, Phys. Rev. B 68, 100508 (2003).

[15] J. Bardeen, L. N. Cooper, and J. R. Schrieffer, Phys. Rev. 108, 1175 (1957).

[16] V. Z. Kresin and S. A. Wolf, Fundamentals of Superconductivity (Plenum Press, New York, 1990), p. 22.

[17] A. Mourachkine, Room-Temperature Superconductivity, (Cambridge International Science Publishing, Cambridge, 2004), pp. 32, 249, 136, 51, 250, 121, 180, 138.

[18] L. P. Gor'kov, Sov. Phys. JETP 7, 505 (1958); ibid 9, 1364 (1959); 10, 998 (1960).

[19] C. Caroli, P. G. de Gennes, and J. Matricon, Phys. Lett. 9, 307 (1964).

[20] F. Gygi and M. Schlüter, Rev. B 43, 7609 (1991). 
[21] Y. Wang and A. H. MacDonalds, Phys. Rev. B 52, R3876 (1995).

[22] M. E. Zhitomirsky and V.-H. Dao, Phys. Rev. B 69, 054508 (2003).

[23] J. G. Rodrogo and S. Viera, Physica C 404, 306 (2004).

[24] E. Boaknin, M. A. Tanatar, J. Paglione, D. G. Hawthorn, R. W. Hill, F. Ronning, M. Sutherland, L. Taillefer, J. Sonier, S. M. Hayden, and J. W. Brill, Physica C (in press).

[25] G. Deutscher, Nature 397, 410 (1999).

[26] A. Mourachkine, preprint cond-mat/9811284 (1998).

[27] A. Mourachkine, Europhys. Lett. 50, 663 (2000).

[28] A. Mourachkine, Europhys. Lett. 55, 559 (2001), and 56 468 (2001).

[29] A. Mourachkine, Supercond. Sci. Technol. 14, 329 (2001).

[30] Yu. I. Latyshev, A. A. Sinchenko, L. N. Bulaevskii, V. N. Pavlenko, and P. Monceau, JETP Lett. 75, 103 (2002).

[31] J. E. Sonier, J. Phys. Cond. Matter (in press), also preprint cond-mat/0404115 (2004).

[32] H. H. Wen, H. P. Yang, S. L. Li, X. H. Zeng, A. A. Soukiassian, W. D. Si, and X. X. Xi, preprint condmat/0305244 (2003). 